\title{
Time-dependent spatial amplitude patterns of harmonic tremor at Arenal volcano, Costa Rica: seismic wave interferences?
}

Javier Almendros ${ }^{1,2}$, Rafael Abella ${ }^{3}$, Mauricio Mora ${ }^{4}$, Philippe Lesage ${ }^{5}$

${ }^{1}$ Instituto Andaluz de Geofísica, Universidad de Granada, 18071 Granada, Spain

${ }^{2}$ Dep. Física Teórica y del Cosmos, Universidad de Granada, 18071

Granada, Spain

${ }^{3}$ Observatorio Geofísico Central, Instituto Geográfico Nacional, 28014

Madrid, Spain

${ }^{4}$ Escuela Centroamericana de Geología, Universidad de Costa Rica, AP

214-2060 San José, Costa Rica

${ }^{5}$ Institut des Sciences de la Terre, Université de Savoie, CNRS, F-73376 Le Bourget-du-Lac, France

Version June 27, 2012, submitted to Bull. Seism. Soc. Am.

Contact info: Javier Almendros, Instituto Andaluz de Geofísica, Universidad de Granada, Campus de Cartuja s/n, 18071 Granada, Spain, tel +34 958 243556, fax +34 958 160907, email alm@iag.ugr.es 
Abstract. Seismograms recorded at the receivers of a small-aperture seismic array usually display very similar waveforms and amplitudes, as a consequence of their close proximity. During the analysis of the volcanic tremor wavefield at Arenal volcano, Costa Rica, we detected significant differences in the amplitudes of harmonic tremor recorded at the stations of a small-aperture $(\sim 210 \mathrm{~m})$ seismic array. The amplitude distributions are geometrically complex and characterized by strong gradients. They occur just for harmonic tremors; any other type of seismic event produces nearly uniform amplitudes across the array. This suggests some relation with harmonic frequency content. Moreover, the spatial amplitude patterns change with time. Some of these observations could be explained by an extreme combination of source, path and site effects. But they could be also produced by interference of different components of the seismic wavefield. We use numerical calculations to investigate the amplitude pattern generated by two interfering plane waves, and are able to reproduce the main features of the observed amplitude patterns. We propose mechanisms that might generate seismic wavefields with multiple components and conclude that interference can explain the complexity and variability of the harmonic tremor wavefield at Arenal volcano. 


\section{Introduction}

Harmonic tremor is a continuous seismo-volcanic signal with a duration of minutes to hours and a spectrum characterized by the presence of several regularly spaced peaks. It has been reported at volcanoes around the world, for example Sakurajima, Japan [Maryanto et al., 2008], Semeru, Indonesia [Schlindwein et al., 1995], Erebus, Antarctica [Rowe et al., 2000], Soufriere Hills, Montserrat [Neuberg et al., 2000], Karymsky, Russia, and Sangay, Ecuador [Johnson and Lees, 2000], Lascar, Chile [Hellweg, 2000], and Arenal, Costa Rica [Benoit and McNutt, 1997; Garces et al., 1998a; Hagerty et al., 2000; Lesage et al., 2006].

Most studies of harmonic tremor are based on data from single seismic stations or distributed networks. They focus mainly on detailed investigations of the harmonic tremor spectrum and its temporal evolution, which constitutes indeed their most striking feature [Schlindwein et al., 1995; Benoit and McNutt, 1997; Hagerty et al., 2000; Lesage et al., 2006; Maryanto et al., 2008]. Some of them also include analyses of wave polarization [Hagerty et al., 2000; Lees et al., 2004; Maryanto et al., 2008], non-linear dynamics [Julian, 2000; Lees and Ruiz, 2008], comparisons between seismic and acoustic observations [Garces et al., 1998a; Hagerty et al., 2000; Johnson and Lees, 2000; Lees et al., 2008], etc. The origin of harmonic tremors is still poorly understood, although feasible models have been proposed. We can mention the resonance of fluid-filled cavities [Chouet, 1992; Benoit and McNutt, 1997], the excitation of conduit walls due to unsteady fluid flow [Julian, 1994, 2000; Rust et al., 2008; Fujita et al., 2011], and the repetition of highly periodic transients originated in the fluid dynamics [Schlindwein et al., 1995; Hellweg, 2000; Johnson and Lees, 2000; Powell and Neuberg, 2003].

In this paper, we investigate the seismic amplitudes of harmonic tremors recorded at the receivers of a dense, small-aperture seismic array deployed at Arenal volcano, Costa Rica. 
The results show the occurrence of complex spatial and temporal patterns, and may shed some light on the nature of the harmonic tremor wavefields.

\section{Harmonic tremors at Arenal volcano}

Arenal is a small stratovolcano located in northwestern Costa Rica (Figure 1). It remained very active during 42 years from 1968 when eruption began, until october 2010, when the volcanic activity suddenly stopped. During this period, volcanic activity was characterized by lava extrusion, strombolian explosions, and gas emissions, at an approximately constant rate [Williams-Jones et al., 2001; Wadge et al., 2006]. The magma composition was almost constant as well, suggesting a steady recharge of the magmatic chamber (Streck et al., 2002). Seismic activity was characterized by a variety of signals including harmonic and spasmodic tremor, explosion quakes, long-period (LP) events, rockfall events and some volcano-tectonic (VT) swarms [Alvarado et al., 1997; Lesage et al., 2006]. Some of the events were accompanied by acoustic waves [Barquero et al., 1992; Garces et al., 1998a; Hagerty et al., 2000].

Harmonic tremor was the most conspicuous seismic signal at Arenal volcano, lasting several hours per day. The fundamental frequencies are generally around 1-2 Hz [Benoit and McNutt, 1997; Hagerty et al., 2000], although they are far from stable. Detailed analyses of the spectral content of Arenal harmonic tremors [Lesage et al., 2006] show the occurrence of a few distinct behaviors: (1) smooth variations of the fundamental frequency (and its overtones) as large as $50 \%$ in time scales of minutes to tens of minutes (frequency gliding); (2) sudden jumps in the fundamental frequency (and its overtones); (3) generation of harmonic tremor following some LP events or explosion quakes and evolution of harmonic tremor into spasmodic tremor and viceversa; and (4) simultaneous presence of several, independent systems of overtones, indicating the activation of multiple tremor sources.

The interpretation of the spectral properties of Arenal harmonic tremor in the framework of different source models and the comparison between seismic and acoustic observations 
hint at a shallow source at the volcano summit [Garces et al., 1998a; Lesage et al., 2006]. Quantitative source locations using multiple seismic arrays [Metaxian et al., 2002] show that the seismogenetic area lays within $600 \mathrm{~m}$ of the active crater. The sources of discrete LP events and explosions are also located in the same area [Alvarado et al., 1997; Hagerty et al., 2000; Metaxian et al., 2002]. Moreover, moment tensor inversions hint to a source at about 100 and $200 \mathrm{~m}$ depth under the crater for tremor and explosions, respectively [Davi et al., 2010, 2012]. Thus the source of Arenal harmonic tremors seems to be linked to the fluid dynamics of the active volcanic conduits located at shallow depths below the crater.

\section{Instruments and data}

In February 2004 a dense, small-aperture seismic array was deployed during 2.5 days (Feb 22 01:00 - Feb 24 14:00) on the western slopes of Arenal volcano, at about $2 \mathrm{~km}$ from the volcano summit (Figure 1). The array was composed by 19 short-period Lennartz LE-3Dlite seismometers, with natural frequency of $1 \mathrm{~Hz}$. We used Reftek 130 dataloggers with a sampling frequency of 100 sps. Synchronism was achieved using GPS time. The seismometers were distributed in a spiral configuration (Figure 1) with an aperture of 210 m. Stations are numbered clockwise from the array center. Stations 1-5 are located near the center and stations 10,13,16, 19 are the farthest to the west, north, east, and south, respectively. Station 13 did not work for the first 20 hours and a few more stations were intermittently up and down from 12:00 to 14:00 on February 22.

The seismic data recorded during the 60-hour interval of array operations reflect the continuous character of the volcanic activity at Arenal volcano. The data contain several ( 200) LP events and different types of volcanic tremor. Harmonic tremor characterized by narrow peaks at equal frequency intervals is conspicuous, covering about $75 \%$ of the time. 


\section{Spatial amplitude patterns at the array stations}

Seismic records from small-aperture arrays usually display similar amplitudes and waveforms. This is a consequence of the close proximity of the array receivers and constitutes the basis of array processing techniques.

Figure 2a shows the waveforms of an LP event recorded at the 19 array stations. We can see that amplitudes are similar at all stations. This is the expected behavior of a plane wavefront propagating in a homogeneous medium. In other words, this is the expected farfield behavior when the effects of attenuation, wavefront distortions due to the topography and other lateral heterogeneities, local velocity anomalies under the stations, etc., are small. In order to quantify the amplitude of the seismic wavefield recorded at the different array channels, we use the RMS of the filtered vertical-component seismograms. We select the 1-4 $\mathrm{Hz}$ band for the analysis, mainly for two reasons: (1) this band contains most of the energy of the LP seismicity and the fundamental peak of harmonic tremors; and (2) wavelengths are large compared with interstation distances, which ensures that the similarity of waveforms is maintained. Figure $2 \mathrm{~b}$ shows the filtered traces (left plot) corresponding to the window highlighted in Figure 2a, and two representations of their RMS: the absolute RMS values for the different array channels (center plot) and the RMS values normalized by their maximum (right plot). The normalized values are plotted as colored circles at the corresponding station locations. Therefore, this plot gives a representation of the spatial amplitude pattern of the seismic wavefield. Normalized RMS values may range from 0 (flat seismogram, dark colors) to 1 (RMS equal to the maximum, light colors). Since RMS values are normalized, there is always at least one white point with normalized RMS of 1 . The question is whether the remaining values are lower or not. In this case, since seismic amplitudes are nearly the same at all stations, the circles are all white, indicating normalized RMS values near 1.

Figure 3a shows a sample of two minutes of vertical-component array data corresponding to a harmonic tremor with a dominant frequency of about $2 \mathrm{~Hz}$. During the first part of 
the window, tremor amplitudes are similar at all stations, just as in the case of the LP event described above. However, a closer look at Figure 3a shows that a minute later the amplitude pattern of harmonic tremor is significantly different. If we compare the two highlighted windows, we find that at station 19 (the southern tip of the array) tremor amplitudes are approximately the same; while at station 1 (located at the array center) the tremor amplitude is clearly larger for the first window. Figure 3b shows the filtered data, absolute RMS, and normalized RMS displayed at the corresponding station locations for the first window highlighted in Figure 3a. The amplitudes are approximately constant throughout the array, as denoted by the flat curve of RMS versus station number, and the light colors of the normalized RMS plot. Figure 3c corresponds to the analysis of the second window highlighted in Figure 3a. In this case, the amplitudes are large in the north and south stations, at the same level than the data shown in Figure 3b. However, amplitudes are quite small around an ESE-WNW band across the array center. For some of them, amplitudes are as low as $20 \%$ of the maximum. The comparison of the right plots in Figures $3 \mathrm{~b}$ and $3 \mathrm{c}$ shows that the wave amplitude distribution varies both in time and in space throughout the array, even if the waveforms remain similar. Therefore, we have to state the conclusion that seismograms recorded at very close stations do not always display similar amplitudes. We underscore that both patterns shown in Figure 3 remain stable for several tens of seconds. They are not random transients, but imply a fundamental change in the harmonic tremor wavefield.

The meaning of the right plot of Figure 3c is further illustrated in Figure 4. Here we show snapshots of the vertical component of the wavefield during half a second for the initial part of the data window shown in Figure 3c. Triangles indicate the positions of the array stations. Black dots above these triangles represent the corresponding vertical velocities at each of the array stations. We have added a triangular mesh to simplify the comparison among snapshots. The black dots are projected on a vertical, NNE-SSW plane, 
approximately perpendicular to the dark band observed in the right plot of Figure 3c. We keep these projections as small gray dots on the following snapshots, in order to illustrate the amplitude span of the vertical motion at each array station. These snapshots illustrated in Figure 4 can be visualized in an animation provided as an electronic supplement to this article. We can see that although vertical velocities oscillate with the same period at all stations, the amplitudes are not the same. Seismograms recorded at stations along the dark band of Figure 3c display small amplitudes compared to the north and south stations. This can be seen clearly in the last snapshot, where the vertical distribution of gray dots spans a larger vertical range for the north and south stations than for stations projected near the center.

In order to visualize the temporal variations of the amplitude patterns at the array for all the dataset (60 hours), we calculate the RMS at every station using a 10-s moving window, sliding $2.5 \mathrm{~s}$ each step. For each time window, we normalize the RMS values dividing by the maximum RMS of the array stations. In this way we remove the effect of temporal variations in the seismogram amplitude and simplify the comparison of spatial amplitude patterns. We obtain 19 time series of normalized RMS, one for each receiver. With these data, we build plots that display the normalized RMS (with a color scale) as a function of time (x-axis) and station number (y-axis). In these plots, vertical sections represent the normalized RMS distributions across the array for particular times. They are just straightened versions of the spatial amplitude patterns. Although we lose the spatial information contained for example in the right panels of Figures $2 \mathrm{~b}$ and $3 \mathrm{~b}, \mathrm{c}$, we can easily compare the amplitude patterns for successive time windows.

Figure 5 shows examples of these plots corresponding to four selected 5 -minute-long data windows, together with the array-averaged spectrograms. Vertical sections where normalized RMS values are close to 1 (light colors) for all stations indicate quasi-uniform seismic amplitude distributions. These are the cases illustrated in Figures $2 \mathrm{~b}$ and $3 \mathrm{~b}$. We can 
identify several instances of uniform amplitudes, marked by arrows in Figure 5. They are related both to LP events and harmonic tremors, as evidenced by the spectrograms. Most interestingly, we observe windows with very different amplitudes across the array. This effect can be recognized by the dark colors (low values of normalized RMS) at some stations. These patterns remain stable from tens of seconds to minutes. The right plots of Figure 5 show the average spatial amplitude patterns observed within the windows marked by dashed lines in the left plots, where amplitude differences are noticeable and stable. These windows are dominated by harmonic tremor, as can be seen in the spectrograms. Dark circles indicate that, at those particular stations, harmonic tremor reaches only a small fraction of the amplitude recorded at other, nearby stations. The patterns are quite diverse, reflecting that they are not linked to any particular site or receiver. There are periods with uniform amplitudes followed by periods with strong amplitude differences among the array stations. At this point, it is very important to underline that the stations with reduced amplitudes are not always the same. Hence, it appears that the spatial amplitude distributions produced by the seismic wavefield at our array are geometrically complex and strongly time-dependent at time scales of minutes.

The presence of strong spatial amplitude gradients at the seismic array is very common in our dataset, and is usually linked to harmonic tremors. Complex amplitude patterns are clearest during harmonic tremors with clean, well-developed overtones and relatively stationary fundamental frequencies. They are least obvious during episodes of fast frequency gliding or when multiple sets of overtones appear simultaneously. Other types of signals (tectonic earthquakes, LP events, spasmodic tremor, even background noise) are recorded with approximately uniform amplitudes at all array receivers (see Figures 2 and 5).

\section{Origin of the complex spatial amplitude pattern of harmonic tremor}

Any mechanism proposed to explain the origin of the amplitude variations detected at the array has to take into account the observations described in the previous section: (1) 
there are large amplitude differences among array stations separated by just 20-200 m, which produces strong amplitude gradients; (2) the spatial amplitude patterns at the array stations are geometrically complex; (3) they are stable at time scales of tens of seconds, but change drastically with time at longer scales; and (4) these patterns are related solely to harmonic tremors and disappear for any other type of seismic events.

\subsection{Source, path, and site effects}

The first two observations above can be explained invoking extreme site and/or path effects. Site effects related to a heterogeneous shallow structure under the array may produce differential amplifications at the array stations [Aki and Ferrazzini, 2000; Almendros et al., 2004; Tramelli et al., 2010]. Site effects at Arenal volcano have been investigated by Mora et al. [2001] and Mora et al. [2006]. They found that the shallow structure under our array site is laterally heterogeneous, as evidenced by a refraction profile and by the different behavior of the $\mathrm{H} / \mathrm{V}$ ratios at nearby stations. They also observed strong variations of the relative amplitude of the harmonic tremor spectral peaks between close stations $(\sim 200 \mathrm{~m})$ of linear arrays located on the east and west flanks of the volcano. Using $\mathrm{H} / \mathrm{V}$ spectral ratios, they were partially able to relate these variations to the shallow velocity structure.

We have also calculated $\mathrm{H} / \mathrm{V}$ spectral ratios using the current array data. Figure 6 displays the $\mathrm{H} / \mathrm{V}$ spectral ratios calculated by using the Geopsy software (www.geopsy.org, e.g. Wathelet et al. [2008]). The distribution of peak frequencies clearly shows two regions. Stations 11 to 16, located at the northern and eastern parts of the array, are characterized by frequencies of $1.6 \mathrm{~Hz}$, while the remainders have frequencies close to $2 \mathrm{~Hz}$. The transition in frequency occurs in a few tens of meters, as can be observed between stations 10 and 11 and between stations 16 and 17 . This is probably related to a strong local heterogeneity in the shallow structure below the array. However, no geological evidence of such heterogeneity is observed at the surface. 
These results indicate that site effects may be partially responsible of the disparate seismic amplitudes observed in the array records. Path effects related to wavefield distortions and seismic energy focusing [Auger et al., 2003; Blacic et al., 2009; Garcia-Yeguas et al., 2011], effect of surface topography [Almendros et al., 2001; Ripperger et al., 2003; Metaxian et al., 2009], etc., may also produce variations of seismic amplitudes among nearby stations. However, since the volcano velocity structure does not change significantly with time (at least at short time scales), site and path effects by themselves are not able to explain the third observation above.

In order to explain the temporal variability of the amplitude patterns, we could hypothesize that they are related to changes in the tremor source location. The tremor source at Arenal volcano is located within a few hundred meters of the summit [Metaxian et al., 2002; Davi et al., 2010, 2012]. There are evidences that point to the activation of different source areas, either sequentially or simultaneously [e.g. Lesage et al., 2006]. In a highly heterogeneous medium, even small changes in source location may produce significantly different ray paths between the source and the receiver. Therefore, we could account for the first three observations by invoking changes in the source location combined with extreme path effects in a highly heterogeneous medium.

But even if we justify the appearance of complex spatial amplitude patterns and why they change with time, we must explain why they occur only for harmonic tremors. Array analyses demonstrate that LP events are have fast first arrivals, what points to a deeper source region. And perhaps the path effects are not as remarkable there. The same applies to VT and regional earthquakes, and even noise. However, spasmodic tremors are generally regarded as a different manifestation of the harmonic tremor source, lacking only its striking regularity [Benoit and McNutt, 1997; Hagerty et al., 2000; Lesage et al., 2006]. Thus the spasmodic tremor source location would be similar to that of harmonic tremor. In this 
context, both harmonic and spasmodic tremors should display complex amplitude patterns, which are not observed. Therefore, we need another explanation.

\subsection{Amplitude patterns produced by interference}

The characteristics of the amplitude distributions at the array stations and the peculiar spectral features of harmonic tremor, the only signal that produces the complex patterns described above, suggest the idea that they might be related to constructive and destructive interference effects.

Two plane waves with different frequencies and different apparent slowness vectors produce an interference pattern characterized by the presence of crests where the amplitude is maximum and nodes where the amplitude is minimum or even no motion is observed at all. The square amplitude $S$ of the wavefield depends on spatial position $\vec{r}$ and time $t$, and is given by

$$
S=A^{2}+B^{2}+2 A B \cos (2 \pi \Delta f t-\Delta \vec{k} \cdot \vec{r}+2 \pi \Phi)
$$

where $A, B$ are the two wave amplitudes, $\Delta \vec{k}$ is the difference of wavenumber vectors of the two plane waves, $\Delta f$ is the difference of frequencies, and $\Phi$ is a number between 0 and 1 that represents an arbitrary phase. Thus the seismic amplitude produced by interference of two plane waves behaves as a plane wave itself, with a frequency given by the difference of frequencies and a wavenumber vector given by the difference of the wavenumber vectors of the interfering waves. The crests corresponding to the maximum amplitude $(A+B)^{2}$ (constructive interference) verify the equation

$$
\Delta \vec{k} \cdot \vec{r}=2 \pi(\Delta f t+\Phi+N)
$$

where $N$ is an integer number. We can see that when the two interfering waves have the same frequency, the amplitude pattern is stationary. When the wave frequencies are slightly different, the amplitudes change slowly with time. In these cases, waveforms display a pulsating (or beating) envelope, a phenomenon that is frequently observed in the raw data 
at different time scales (Figure 7). The variations are faster when the two wave frequencies are more different. In the limit, when the frequencies are not similar, the amplitude beats occur several times during the averaging window, and the amplitude variations cannot be observed any more.

We use synthetic data to calculate the spatial amplitude pattern generated at the array stations by the interference of two harmonic tremor signals. We use two models of harmonic tremor. The simplest model considers a sine function and represents the fundamental mode of harmonic tremor. The second model is based on the periodic repetition of a transient signal [Schlindwein et al., 1995; Hagerty et al., 2000; Lesage et al., 2006]. We use a Ricker wavelet to represent the seismic signature of the source. In this case the synthetic tremor signal is more realistic, and reproduces not only the fundamental frequency but the overtones as well.

Figure 8 shows an example of the spatial amplitude pattern generated by the interference of two plane waves. The waves have the same frequencies of $2.5 \mathrm{~Hz}$. They propagate with apparent slownesses of 0.9 and $1.4 \mathrm{~s} / \mathrm{km}$ and azimuths of 225 and $280^{\circ} \mathrm{N}$ (back-azimuths of 45 and $\left.100^{\circ} \mathrm{N}\right)$. The resulting destructive interference fringes are linear, and perpendicular to the difference of wavenumber vectors, as inferred from Equation (2). The spatial amplitude pattern resulting from this particular case of wave interference is similar to the examples shown in Figures 3c and 5d, in the sense that amplitudes are large in the northern and southern stations and small along an ESE-WNW band crossing the array center. The pattern is a bit more complex when using the repetitive-source tremor model, due to the presence of shorter-wavelength overtones. The reduced amplitudes are mostly due to interference of the fundamental mode. At the interference nodes, the fundamental mode would be basically filtered out. This filtering effect is clear in the synthetics of Figure 8c, but can also be observed in the real data shown in Figure 3c. 
Interference is able to justify the main features of the spatial amplitude patterns. It explains the strong gradients and complex geometries (at least partially, more work should be done in this direction). Moreover, it provides a reason why we only find these patterns for harmonic tremors. Indeed, stable interference patterns require narrow-band signals; wide band signals do not produce interference patterns. Finally, in order to explain the temporal variations of the spatial amplitude patterns we have to hypothesize that different waves with varying apparent slowness vectors can be simultaneously present in the wavefield. This possibility is discussed below.

\subsection{Possible causes of interference at Arenal volcano}

Interference requires the presence of multiple, simultaneous narrow-band seismic waves. We have analyzed the present dataset using frequency-slowness array methods. These techniques allow for the identification of different, simultaneous components of the seismic wavefield recorded at the array. In this case, we identify several periods when multiple components are present in the wavefield. All of them coincide with periods dominated by harmonic tremors. The behavior of these wavefield components varies along the dataset. There are examples of waves with similar apparent slownesses but quite different back-azimuths in the range $40-120^{\circ} \mathrm{N}$, which do not usually coincide with the array-crater direction; waves with similar azimuths but different apparent slownesses in the range from 0.5 up to $3 \mathrm{~s} / \mathrm{km}$; and waves with both different apparent slownesses and different azimuths. In some occasions, simultaneous components may be arriving with propagation azimuths that differ by up to $\sim 80^{\circ}$.

Figure 9 shows two examples of results obtained using the MUSIC algorithm $[$ Schmidt, 1986; Goldstein and Archuleta, 1987] with our array data. In Figure 9a we display the results for the same data used for Figure 3. The two gray bands indicate the 5 -s windows detailed in Figures 3b,c. We recall that we have found different behaviors in terms of amplitude distributions for these two windows. The first one is characterized by quasi- 
uniform amplitudes at all array stations, while the second one presents large amplitude differences across the array. Looking at the frequency-slowness results, we notice a single solution for the first window, with apparent slowness of 0.6-0.9 s/km and back-azimuth of 55$65^{\circ} \mathrm{N}$. On the contrary, around the second window, we are able to determine the presence of two components in the harmonic tremor wavefield. They propagate with apparent slownesses of $0.8-1.0$ and $1.2-1.5 \mathrm{~s} / \mathrm{km}$ and back-azimuths of $85-110$ and $35-50^{\circ} \mathrm{N}$, respectively. These apparent slowness vectors were selected for the synthetic example of Figure 8.

The presence of two wavefield components is even clearer in Figure 9b. Around the center of the window, and for about one minute, we find two stable trends corresponding to the main and secondary peaks of the MUSIC estimate (gray and white diamonds, respectively). The main wavefield component propagates with apparent slowness of 0.6-0.9 s/ $\mathrm{km}$ and back-azimuth of $90-120^{\circ} \mathrm{N}$. The secondary component propagates with apparent slowness of $2.5-2.8 \mathrm{~s} / \mathrm{km}$ (very slow) and back-azimuth of $30-45^{\circ} \mathrm{N}$.

The quality of the estimates (bottom plots) shows that in the second window highlighted in Figure 9a the wavefield is composed by two waves with similar power and coherency. In Figure 9b one of the components is clearly dominant, as demonstrated by the quality differences between the estimates for the main and secondary peaks.

The presence of multiple components in the wavefield can be generated by a number of phenomena, including source and path effects. The first hypothesis is that these waves are a consequence of multiple, simultaneous harmonic tremor sources. In fact, several studies evidence the occurrence of multiple harmonic tremor sources at Arenal volcano [Benoit and McNutt, 1997; Hagerty et al., 2000; Lesage et al., 2006]. At some periods, different sets of overtones evolving independently are clearly detected. Each set has to be related to a different tremor source. Moreover, Lesage et al. [2006] documented the simultaneous activity of two craters at the Arenal volcano summit, and linked them to a double tremor source related to shallow conduit branching. These sources are located at shallow depths below 
the crater [e.g. Metaxian et al., 2002]. But the results of the frequency-slowness analysis cannot be easily reconciled with multiple harmonic tremor sources located at shallow depths below the crater area. The crater is small compared to the distance to the seismic array (of about $2 \mathrm{~km}$ ). The expected propagation azimuth at the array site for a wavefield generated by a source located in the crater can be far from the summit-array direction, up to tens of degrees. This is a consequence of the deviations produced by the heterogeneous velocity structure, topography, etc. [e.g. Garcia-Yeguas et al., 2011]. However, the waves generated by two sources located at the crater area should in principle follow similar paths through the medium and reach the array site with similar azimuths, even if different from the arraysummit direction, and similar apparent slownesses. In such a case, the wavenumber vectors would be similar as well. Thus the interference pattern would have a long wavelength and the amplitude differences among array stations would not be noticeable.

There are two options to overcome this situation. First, we might consider the possibility of two harmonic tremor sources occurring at two distant source regions within the volcano. One of them would be located in the crater area, as reported by several authors, and the other somewhere else. The problem is that there are no evidences whatsoever of such a secondary tremor source. Alternatively, we may picture a volcano with a dramatically heterogeneous velocity structure. In this case, seismic rays could follow quite different paths even for nearby sources, and this could explain the presence of wavefield components with different apparent slowness vectors at the array site.

On the other hand, multiple wavefield components can be generated by path effects. For example, the presence of efficient reflectors in the medium would generate secondary wavefields that would interfere with the direct radiation from a single source. Other structures may contribute to split the wavefield, for example a low-velocity, high-attenuation body located between the source and the array. Similar effects could be produced by scattering by topography and other velocity heterogeneities [La Rocca et al., 2001; Almendros et al., 
2001, 2002; Metaxian et al., 2009]. The secondary wavefield would be characterized by an apparent slowness vector that depends mainly on the scatterer location relative to the source (crater) and the array. Another possibility comes from the superposition of surface wave modes. These modes travel with different apparent slowness vectors (different velocities) and can contribute to the variability of the seismic amplitudes at the array stations.

Whatever mechanism producing the observed interferences, the temporal changes of the amplitude pattern show that a time varying process is involved in the phenomena. A first cause of variability that can be considered is the coupling between seismic and acoustic waves. The propagation of seismic waves with apparent slowness close to $3 \mathrm{~s} / \mathrm{km}$ (Figure $9 \mathrm{~b}$ ) suggests a link with the propagation of acoustic waves in the atmosphere. The time-dependent spatial amplitude patterns related to harmonic tremors at Arenal volcano could be produced by interference between the seismic wavefield radiated by a single tremor source and the associated acoustic wavefield. Acoustic waves in the atmosphere have a great variability in terms of propagation azimuths, due to continuous changes in atmospheric conditions, specially the wind strength and direction [Garces et al., 1998b; Le Pichon et al., 2005; Matoza et al., 2007].

However, a number of issues are raised. The most important is that, in general, acoustic waves in the atmosphere are not very efficient in producing seismic waves in the ground. For example, typical values of the transmission factor for normal incidence are smaller than 0.1\%. Matoza et al. [2009] analyzed the acoustic observations at St. Helens and modeled the interactions between the solid surface and the air. They found evidences of seismic energy radiated into the atmosphere as acoustic waves, but not the opposite. Kitov et al. [1997] investigated acoustic and seismic signals from atmospheric and near-surface bomb blasts. They documented energy conversion processes between acoustic waves in the atmosphere and seismic waves in the ground. These processes are most efficient when the surface wave velocity is similar to the apparent velocity of the acoustic waves. This condition 
is likely achieved at Arenal and most volcanic areas, given the presence of slow shallow layers with velocities near the sound speed in the air. At Arenal volcano, some large explosions produce ground-coupled acoustic waves that are recorded with significant amplitudes in the seismograms [Hagerty et al., 2000]. But this might not be the case for the harmonic tremors analyzed here, which display much smaller amplitudes.

Thus, it appears more likely that the temporal variations of the amplitude patterns are related to source variability. Indeed, several studies have pointed that the source and surrounding medium is constantly changing at different time scales [Cole et al., 2005; Wadge et al., 2006; Lesage et al., 2006; Valade et al., 2012]. In particular, an integrated analysis of seismic and Doppler radar recordings of mild explosions, degassing events, and tremor demonstrated the strong variability of these types of signal and the lack of correlation between them [Valade et al., 2012]. These authors propose a source model for explosions and tremor whereby more or less ash-laden gas flow is controlled by the opening and closing

of fractures in the highly viscous cap atop the conduit. The constantly varying state of the lava cap and the fractures produces non repeatable source conditions that can yield rapid changes in the seismic wave radiation pattern, amplitude and frequency as well as fluctuations in the location and depth of the hypocenter. Combined with multiple path and site effects due to topography and highly heterogeneous structure, even slight variations at the source may result in large modifications of the wavefield which are thus reflected in the amplitude pattern evolution.

\section{Conclusions}

We use data from a dense, small-aperture seismic array to show that seismic amplitudes during harmonic tremor episodes at Arenal volcano display complex spatial distributions. We have found strong amplitude variations among array receivers separated just a few tens of meters. The amplitude patterns may remain stable for some tens of seconds, but change 
drastically with time at longer scales. This phenomenon is observed just for harmonic tremors and disappears for any other type of seismic event.

We discuss the source, path, and site effects reported at Arenal volcano. Our observations could be explained invoking a combination of extreme source, path, and site effects. However it would produce similar effects on other types of seismic events. Thus we conclude that such a combination is not very likely to happen. Alternatively, we propose that the complex spatial amplitude patterns could be related to interference of multiple components of the seismic wavefield. This phenomenon explains the characteristics of the spatial amplitude patterns, including their mere existence, their temporal variations and dependence on the harmonic content of the signal. We propose that the interfering waves might originate by a number of mechanisms, for example the simultaneous occurrence of multiple harmonic tremor sources, the superposition of a tremor wavefield from a single source with a scattered wavefield, and the interaction between seismic and acoustic energy radiated by a single tremor source at the volcano.

Our observations document the generation of complex amplitude patterns during harmonic tremors at Arenal volcano. Our interpretation in terms of interference phenomena explains also other observations of strong variations in waveform and relative amplitude of spectral peaks between close stations at different locations of Arenal volcano [Mora et al., 2001, 2006]. An interesting question is whether these patterns are a common feature elsewhere. Unfortunately most seismic studies on volcanoes rely on distributed networks of seismometers, which cannot be used to analyze short-scale variations in the wavefield amplitude. To solve this question, studies of the amplitude distribution within dense seismic arrays should be performed at other volcanoes producing harmonic tremors similar to Arenal. Interference between seismic and acoustic wavefields originated by harmonic tremors should be also further investigated. Experiments combining seismic and infrasonic instruments would be highly desirable. 
In any case, there is more information in our data, in different aspects that we have not addressed so far. The present analysis will be completed with the study of the three components of motion and a wavefield decomposition using array methods, both of them already in progress. The results may help us understand the complex behavior of the harmonic tremor wavefield at Arenal volcano.

Data and resources. Seismograms used in this study were collected during a short experiment carried out by the Observatorio Sismológico y Vulcanológico de Arenal y Miravalles (OSIVAM-ICE) in February 2004. Data will be available after exploitation and can be obtained through request to Taylor Castillo Waldo (wtaylor@ice.go.cr). Some plots and analyses were made using MATLAB (http://www.mathworks.com).

Acknowledgments. We thank Dr. Chalotte Rowe and two anonymous reviewers for their useful comments and suggestions. We are grateful to ICE for supporting the logistical work and the data acquisition. We are also grateful to the Arenal Conservation Area staff for the facilities provided during the field work. The financial support from the projects No 113A4-501 and 113-A6-503 from University of Costa Rica, Ministry of Science and Technology (MICIT) and National Council for Scientific and Technological Research (CONICIT) is acknowledged. Partial support was also provided by projects CTM2010-11740 and CGL201129499-C02-01 of the Spanish Ministry of Science and Innovation.

\section{References}

Aki, K., Ferrazzini, V. (2000), Seismic monitoring and modeling of an active volcano for prediction, J. Geophys. Res., 105(B7), 16617-16640.

Almendros, J., Chouet, B., Dawson, P. (2001), Spatial extent of a hydrothermal system at Kilauea Volcano, Hawaii, determined from array analyses of shallow long-period seismicity

- 1. Method, J. Geophys. Res., 106(B7), 13565-13580, doi:10.1029/2001JB000310. 
Almendros, J., Chouet, B., Dawson, P., Huber, C. (2002), Mapping the sources of the seismic wavefield at Kilauea Volcano, Hawaii, using data recorded on multiple seismic antennas, Bull. Seism. Soc. Am., 92, 2333-2351, doi:10.1785/0120020037.

Almendros, J., Luzon, F., Posadas, A. (2004), Microtremor analyses at Teide volcano (Canary Islands, Spain): Assessment of natural frequencies of vibration using timedependent horizontal-to-vertical spectral ratios, Pure Appl. Geophys., 161(7), 1579-1596, doi:10.1007/s00024-004-2522-5.

Alvarado, G. E., Taylor, W., Ohmberger, M., Soto, G., Madrigal, L. (1997), First observations of volcanic seismicity at Arenal volcano (Costa Rica) using a new three-component seismic digital network, Boletín del Observatorio Sismológico y Vulcanológico de Arenal y Miravalles, 15-16, 11-45.

Auger, E., Virieux, J., Zollo, A. (2003), Locating and quantifying migration and ava analysis of reflected and converted waves: An application to the Mt. Vesuvius volcano, Geophys. J. Int., 152, 486-496, doi:10.1046/j.1365-246X.2003.01864.x.

Barquero, R., Alvarado, G. E., Matumoto, T. (1992), Arenal volcano (Costa Rica) premonitory seismicity, in Volcanic Seismology, IAVCEI Proceedings in Volcanology, vol. 3, edited by P. Gasparini, R. Scarpa, and K. Aki, pp. 84-96, Springer-Verlag.

Benoit, J. P., McNutt, S. R. (1997), New constraints on source processes of volcanic tremor at Arenal volcano, Costa Rica, using broadband seismic data, Geophys. Res. Lett., 24(4), $449-452$.

Blacic, T. M., Latorre, D., Virieux, J., Vassallo, M. (2009), Converted phases analysis of the Campi Flegrei caldera using active seismic data, Tectonophysics, 470, 243-256, doi: 10.1016/j.tecto.2008.12.006.

Chouet, B. (1992), A seismic model for the source of long-period events and harmonic tremor, in Volcanic Seismology, IAVCEI Proceedings in Volcanology, vol. 3, edited by P. Gasparini, R. Scarpa, and K. Aki, pp. 133-156, Springer-Verlag. 
Cole, P. D., Fernandez, E., Duarte, E., Duncan, A. M. (2005), Explosive activity and generation mechanisms of pyroclastic flows at Arenal volcano, Costa Rica between 1987 and 2001, Bull. Volcan., 67, 695-716, doi:10.1007/s00445-004-0402-6.

Davi, R., O’Brien, G. S., Lokmer, I., Bean, C. J., Lesage, P., Mora, M. M. (2010), Moment tensor inversion of explosive long period events recorded on Arenal volcano, Costa Rica, constrained by synthetic tests, J. Volcan. Geotherm. Res, 194, 189-200, doi: 10.1016/j.jvolgeores.2010.05.012.

Davi, R., O’Brien, G. S., Barros, L. D., Lokmer, I., Bean, C. J., Lesage, P., Mora, M. M., Soto, G. J. (2012), Seismic source mechanisms of tremor recorded on Arenal volcano, Costa Rica, retrieved by waveform inversion, J. Volcan. Geotherm. Res, 213-214, 1-13, doi:10.1016/j.jvolgeores.2011.10.008.

Fujita, E., Araki, K., Nagano, K. (2011), Volcanic tremor induced by gas-liquid twophase flow: Implications of density wave oscillation, J. Geophys. Res., 116, B09201, doi: 10.1029/2010JB008068.

Garces, M. A., Hagerty, M. T., Schwartz, S. Y. (1998a), Magma acoustics and time-varying melt properties at Arenal volcano, Costa Rica, Geophys. Res. Lett., 25, 2293-2296.

Garces, M. A., Hansen, R. A., Lindquist, K. G. (1998b), Traveltimes for infrasonic waves propagating in a stratified atmosphere, Geophys. J. Int., 135, 255-263.

Garcia-Yeguas, A., Almendros, J., Abella, R., Ibaez, J. M. (2011), Quantitative analysis of seismic wave propagation anomalies in azimuth and apparent slowness at Deception Island volcano (Antarctica) using seismic arrays, Geophys. J. Int., 184, 801-815, doi: 10.1111/j.1365-246X.2010.04864.x.

Goldstein, P., Archuleta, R. J. (1987), Array analysis of seismic signals, Geophys. Res. Lett., 14(1), 13-16.

Hagerty, M. T., Schwartz, S. Y., Garces, M. A., Protti, M. (2000), Analysis of seismic and acoustic observations at Arenal Volcano, Costa Rica, 1995-1997, J. Volcan. Geotherm. 
Res., 101(1-2), 27-65.

Hellweg, M. (2000), Physical models for the source of Lascar's harmonic tremor., J. Volcan. Geotherm. Res., 101(1-2), 183-198.

Johnson, J. B., Lees, J. M. (2000), Plugs and chugs-seismic and acoustic observations of degassing explosions at Karymsky, Russia and Sangay, Ecuador, J. Volcan. Geotherm. Res., 101(1-2), 67-82.

Julian, B. R. (1994), Volcanic tremor: nonlinear excitation by fluid flow, J. Geophys. Res., 99(B6), 11859-11877.

Julian, B. R. (2000), Period doubling and other nonlinear phenomena in volcanic earthquakes and tremor, J. Volcan. Geotherm. Res., 101(1-2), 19-26.

Kitov, I. O., Murphy, J. R., Kusnetsov, O. P., Barker, B. W., Nedoshivin, N. I. (1997), An analysis of seismic and acoustic signals measured from a series of atmospheric and near-surface explosions, Bull. Seism. Soc. Am., 87, 1553-1562.

La Rocca, M., Del Pezzo, E., Simini, M., Scarpa, R., De Luca, G. (2001), Array analysis of seismograms from explosive sources: evidence for surface waves scattered at the main topographical features, Bull. Seism. Soc. Am., 91(2), 219-231.

Le Pichon, A., Blanc, E., Drob, D., Lambotte, S., Dessa, J. X., Lardy, M., Bani, P., Vergniolle, S. (2005), Infrasound monitoring of volcanoes to probe high-altitude winds, J. Geophys. Res., 110, D13106, doi:10.1029/2004JD005587.

Lees, J. M., Ruiz, M. (2008), Non-linear explosion tremor at Sangay Volcano, Ecuador, J. Volcan. Geotherm. Res, 176, 170-178.

Lees, J. M., Gordeev, E. I., Ripepe, M. (2004), Explosions and periodic tremor at Karymsky volcano, Kamchatka, Russia, Geophys. J. Int., 158, 1151-1167.

Lees, J. M., Johnson, J. B., Ruiz, M., Troncoso, L., Welsh, M. (2008), Reventador volcano 2005: Eruptive activity inferred from seismo-acoustic observation, J. Volcan. Geotherm. Res, 176, 179-190. 
Lesage, P., Mora, M. M., Alvarado, G. E., Pacheco, J., Metaxian, J. P. (2006), Complex behavior and source model of the tremor at Arenal volcano, Costa Rica, J. Volcan. Geotherm. Res., 157, 49-59.

Maryanto, S., Iguchia, M., Tameguri, T. (2008), Constraints on the source mechanism of harmonic tremors based on seismological, ground deformation, and visual observations at Sakurajima volcano, Japan, J. Volcan. Geotherm. Res, 170, 198-217.

Matoza, R. S., Hedlin, M. A. H., Garces, M. A. (2007), An infrasound array study of Mount St. Helens, J. Volcan. Geotherm. Res., 160, 249-262, doi:10.1016/j.jvolgeores.2006.10.006.

Matoza, R. S., Garces, M. A., Chouet, B. A., D’Auria, L., Hedlin, M. A. H., De GrootHedlin, C., Waite, G. P. (2009), The source of infrasound associated with long-period events at Mount St. Helens, J. Geophys. Res., 114, B04305, doi:10.1029/2008JB006128.

Metaxian, J. P., Lesage, P., Valette, B. (2002), Locating sources of volcanic tremor and emergent events by seismic triangulation: Application to Arenal volcano, Costa Rica, J. Geophys. Res., 107(B10), 2243, doi:10.1029/2001JB000559.

Metaxian, J. P., OBrien, G. S., Bean, C. J., Valette, B., Mora, M. (2009), Locating volcanoseismic signals in the presence of rough topography: wave simulations on Arenal volcano, Costa Rica, Geophys. J. Int., 179, 1547-1557.

Mora, M. M., Lesage, P., Dorel, J., Bard, P. Y., Metaxian, J. P., Alvarado, G. E., Leandro, C. (2001), Study of seismic site effects using H/V spectral ratios at Arenal volcano, Costa Rica, Geophys. Res. Lett., 28(15), 2991-2994.

Mora, M. M., Lesage, P., Valette, B., Alvarado, G. E., Leandro, C., Metaxian, J. P., Dorel, J. (2006), Shallow velocity structure and seismic site effects at Arenal volcano, Costa Rica, J. Volcan. Geotherm. Res., 152, 121-139.

Neuberg, J., Luckett, R., Baptie, B., Olsen, K. (2000), Models of tremor and low-frequency earthquake swarms on Montserrat, J. Volcan. Geotherm. Res., 101(1-2), 83-104. 
Powell, T. W., Neuberg, J. (2003), Time dependent features in tremor spectra, J. Volcan. Geotherm. Res., 128(1-3), 177-185.

Ripperger, J., Igel, H., Wasserman, J. (2003), Seismic wave simulation in the presence of real volcano topography, J. Volcan. Geotherm. Res., 128(1-3), 31-44.

Rowe, C., Aster, R. C., Kyle, P. R., Dibble, R., Schlue, J. (2000), Seismic and acoustic observations at Mount Erebus volcano, Ross Island, Antarctica, 1994-1998, J. Volcan. Geotherm. Res., 101(1-2), 105-128.

Rust, A. C., Balmforth, N. J., Mandre, S. (2008), The feasibility of generating low-frequency volcano seismicity by flow through a deformable channel, in Fluid motions in volcanic conduits: A source of seismic and acoustic signals, Geological Society Special Publications, vol. 307, edited by S. J. Lane and J. S. Gilbert, pp. 45-56, Geologycal Society of London. Schlindwein, V., Wassermann, J., Scherbaum, F. (1995), Spectral analysis of harmonic tremor signals at Mt. Semeru Volcano, Indonesia, Geophys. Res. Lett., 22, 1685-1688.

Schmidt, R. (1986), Multiple emitter location and signal parameter estimation, IEEE Trans. Ant. Prop., 34(3), 276-280.

Tramelli, A., Galluzzo, D., Del Pezzo, E., Di Vito, M. A. (2010), A detailed study of the site effects in the volcanic area of Campi Flegrei using empirical approaches, Geophys. J. Int., 182, 1073-1086, doi:10.1111/j.1365-246X.2010.04675.x.

Valade, S., Donnadieu, F., Lesage, P., Mora, M. M., Harris, A., Alvarado, G. E. (2012), Explosion mechanisms at Arenal volcano, Costa Rica: An interpretation from integration of seismic and Doppler radar data, J. Geophys. Res., 117, B01309, doi: 10.1029/2011JB008623.

Wadge, G., Oramas-Dorta, D., Cole, P. D. (2006), The magma budget of Volcán Arenal, Costa Rica from 1980 to 2004, J. Volcan. Geotherm. Res, 157, 60-74.

Wathelet, M., Jongmans, D., Ohrnberger, M., Bonnefoy-Claudet, S. (2008), Array performances for ambient vibrations on a shallow structure and consequences over Vs inversion, 
J. Seism., 12, 1-19, doi:10.1007/s10950-007-9067-x.

Williams-Jones, G., Stix, J., Heiligmann, M., Barquero, J., Fernandez, E., Gonzalez, E. D. (2001), A model of degassing and seismicity at Arenal volcano, Costa Rica, J. Volcan. Geotherm. Res., 108(1-4), 121-139. 
Figure 1. Location of the seismic array about $2 \mathrm{~km}$ west of the Arenal volcano summit. The insets show the situation of Arenal volcano in Costa Rica (left) and the configuration of the seismic array (right).

Figure 2. (a) Unfiltered vertical-component seismograms from the 19 array stations, showing 1 minute of data containing an LP event. The gray band marks the window shown in b. (b) Filtered traces from the 19 array stations (left), absolute RMS plotted versus station number (center), and normalized RMS displayed at the station positions (right). The labels at the top left of the seismogram plots indicate the origin times of the windows and the data durations. The vertical scales represent velocity counts.

Figure 3. (a) Unfiltered vertical-component seismograms from the 19 array stations, showing 2 minutes of data containing a sample of harmonic tremor. The gray bands mark the windows shown in b and c. (b) Filtered traces from the 19 array stations (left), absolute RMS plotted versus station number (center), and normalized RMS displayed at the station positions (right). (c) Same than (b) for the second window shown in (a). The labels at the top left of the seismogram plots indicate the origin times of the windows and the data durations. The vertical scales represent velocity counts. 
Figure 4. Snapshots of the vertical ground motion recorded at the array during $0.55 \mathrm{~s}$ at the start of the window shown in Figure 3c. The time indicated at the right top of each panel represents the time elapsed since 2004-02-24 08:50:15. The interval between snapshots is $0.05 \mathrm{~s}$ (5 samples). The vertical scale indicates velocity counts. Tick marks in the horizontal planes are spaced $100 \mathrm{~m}$. Triangles indicate the positions of the array stations. Black dots above these triangles represent the corresponding vertical velocities in the z-axis scale. They are connected by a mesh (gray lines) to help visualize a moving surface. White dots are projections of the black dots on a vertical, NNE-SSW plane (see sample dashed lines for station 19). These projections on the vertical plane are plotted in all successive snapshots as gray dots to indicate the spans of the vertical velocities at the array stations. Dashed lines in the vertical plane of the bottom right plot represent the envelope of the vertical ground motion. An animation of these wavefield snapshots is available in the electronic supplement of this article.

Figure 5. Temporal variations of the spectral content and spatial amplitude patterns along four selected 5-minute data windows. For each panel, we show the array-averaged spectrogram (top left), and a representation of the normalized RMS of the seismograms versus time and station number (bottom left). The arrows indicate periods with nearly uniform amplitudes across the array. The right plot shows the average normalized RMS for the time periods indicated by the dashed lines, displayed at the station positions. The color scale shown is the same for the right and bottom left plots. The box and two gray bands that can be seen behind the left plots in (d) indicate the data shown in Figure 3a and the windows zoomed in Figures 3b,c. The labels at the top left of the spectrograms indicate the origin times of the windows and the data durations.

Figure 6. Representation of the resonance frequency of the shallow structure estimated by the $\mathrm{H} / \mathrm{V}$ spectral ratio method for the stations of the seismic array. Circles are shown at the corresponding station locations. Circle areas are proportional to $f-f_{0}$, where $f$ is the resonance frequency and $f_{0}$ is $1.5 \mathrm{~Hz}$. The dashed line indicates a rough limit between two regions with clearly distinct frequencies of $\sim 1.6 \mathrm{~Hz}$ (north) and $\sim 2 \mathrm{~Hz}$ (south). 
Figure 7. Examples of harmonic tremors recorded at one of the seismic array receivers (station 15, see Figure 1), and displaying pulsating envelopes at different time scales. The labels at the top left of the seismogram plots indicate the origin times of the windows and the data durations. The vertical scales represent velocity counts.

Figure 8. Calculation of the spatial amplitude distribution of a complex wavefield generated by interference of two synthetic harmonic tremors. (a) Sketch of the selected wave parameters, corresponding to two plane waves with the same amplitude, the same fundamental frequency of $2.5 \mathrm{~Hz}$, apparent slownesses of 0.9 and $1.4 \mathrm{~s} / \mathrm{km}$, and propagation azimuths of 225 and $280^{\circ} \mathrm{N}$. These parameters are taken from the results of the wavefield decomposition (Figure 9a) during the period shown in Figure 3c. $\vec{k}_{1}$ and $\vec{k}_{2}$ are the two wavenumber vectors, and $\Delta \vec{k}$ is the difference of wavenumbers. Wavefronts are represented by solid and dashed parallel lines. The thick dotted gray line shows the direction of crests and nodes resulting from interference, which is perpendicular to $\Delta \vec{k}$ (see Equation 2). (b) Synthetic seismograms generated at the array stations (left), absolute RMS (center), and spatial distribution of the normalized RMS (right) obtained with the sine wave model. (c) Same than (b) for the repetitive source model. The panels in (b) and (c) are the same than in Figures 2b and 3b,c. However, in the right plot we show the normalized RMS not only for the array stations but for the whole medium, as calculated from the synthetics with a spatial grid of $5 \mathrm{~m}$. 
Figure 9. Two examples of the results of the frequency-slowness analysis for 2-minute long data windows, showing the presence of multiple wave components in the wavefield. From top to bottom, we display the seismograms, apparent slowness, back-azimuth, and quality of the solutions (i.e. normalized MUSIC power). Gray and white diamonds correspond to the main and secondary peaks, respectively. The dashed lines in the back-azimuth plots indicate the direction towards the volcano. The data shown in (a) is the same than in Figure 3a. The two vertical gray bands indicate the windows zoomed in Figures $3 \mathrm{~b}$ and $3 \mathrm{c}$. The labels at the top left of the seismogram plots indicate the origin times of the windows and the data durations. The vertical scales represent velocity counts. 


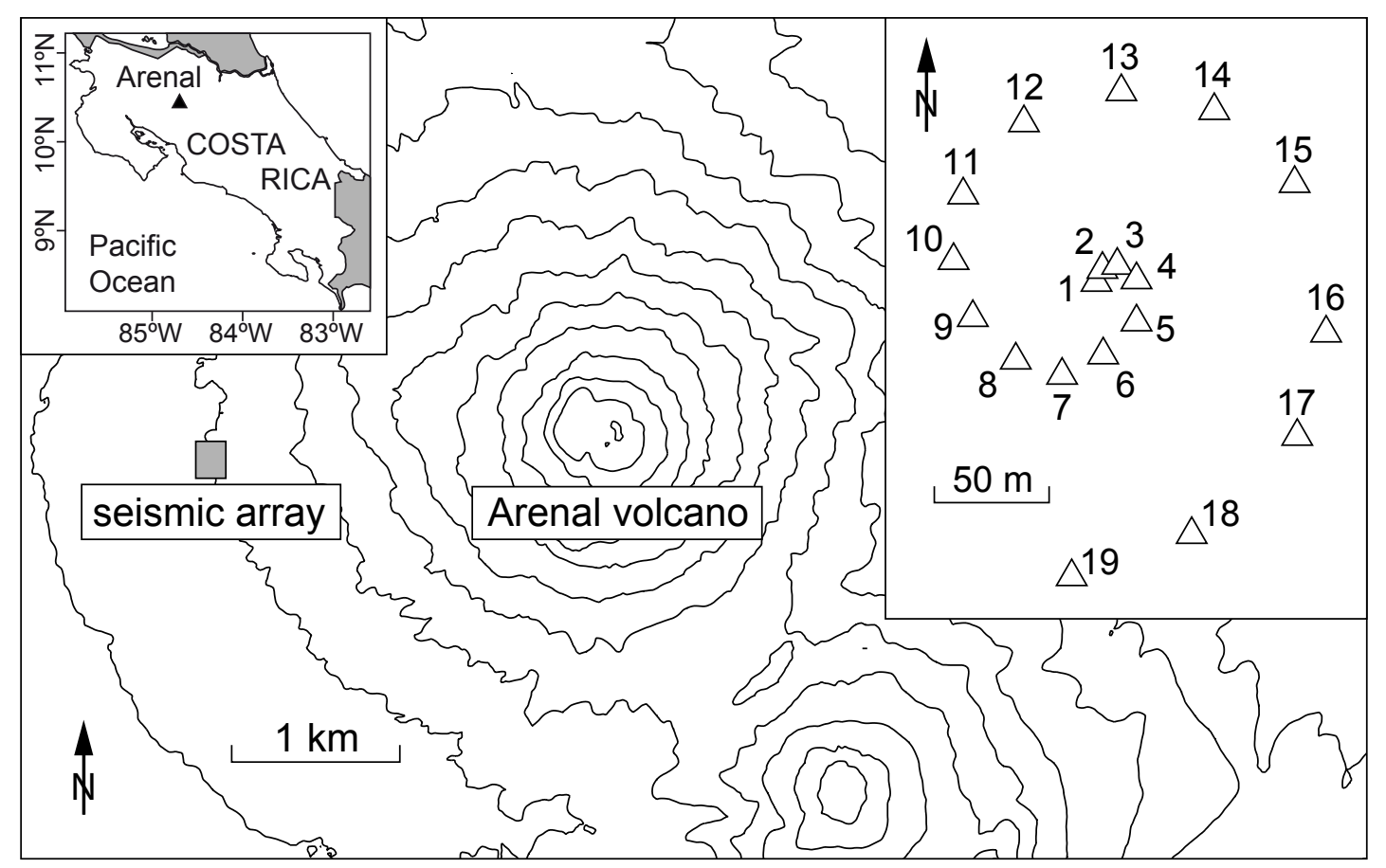

Almendros et al 2012 - Figure 1 
(a) 2004-02-23 22:03:10 $\langle 60 \mathrm{~s}\rangle$

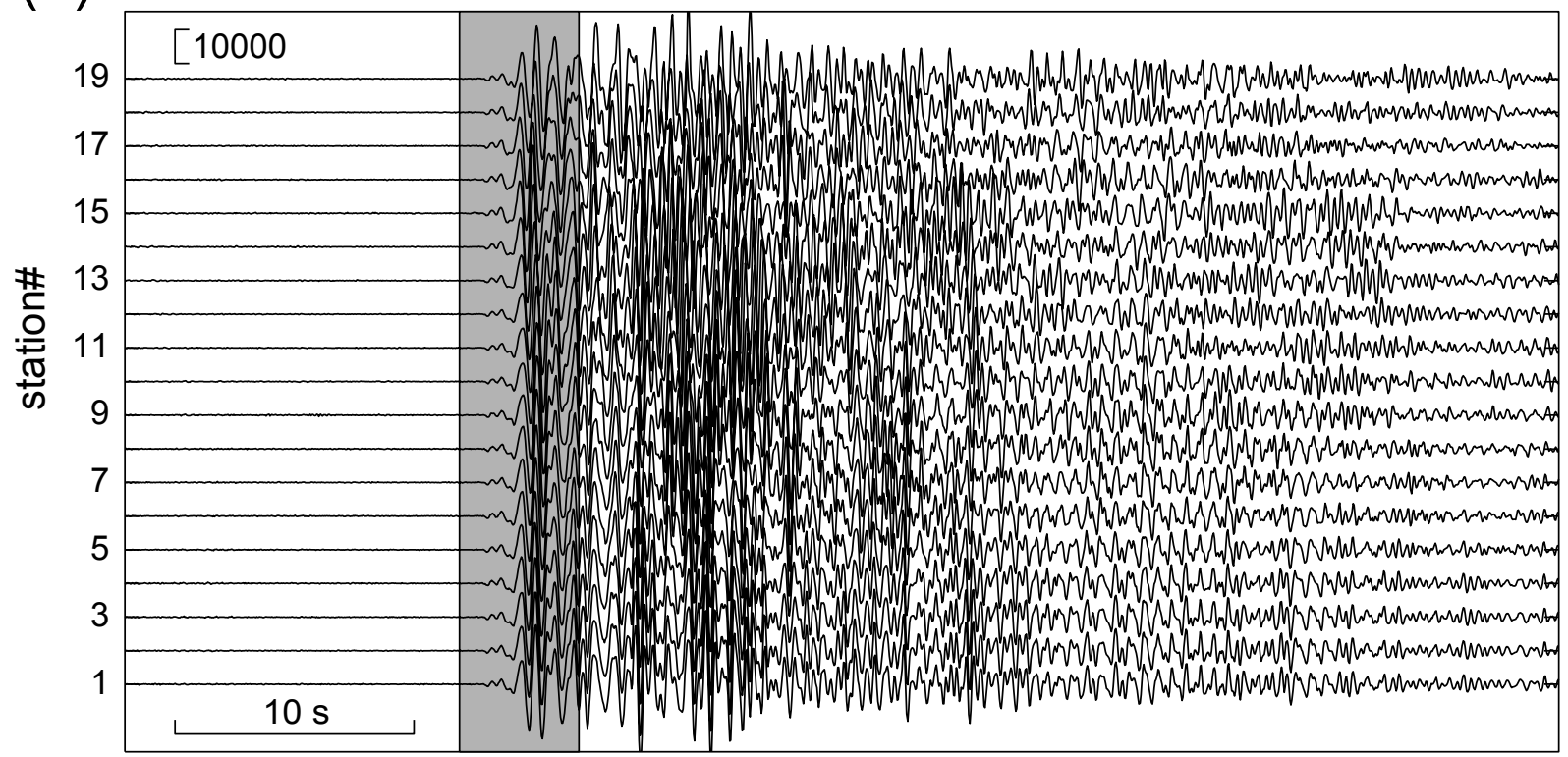

(b) 2004-02-23 22:03:24 $\langle 5 \mathrm{~s}\rangle$

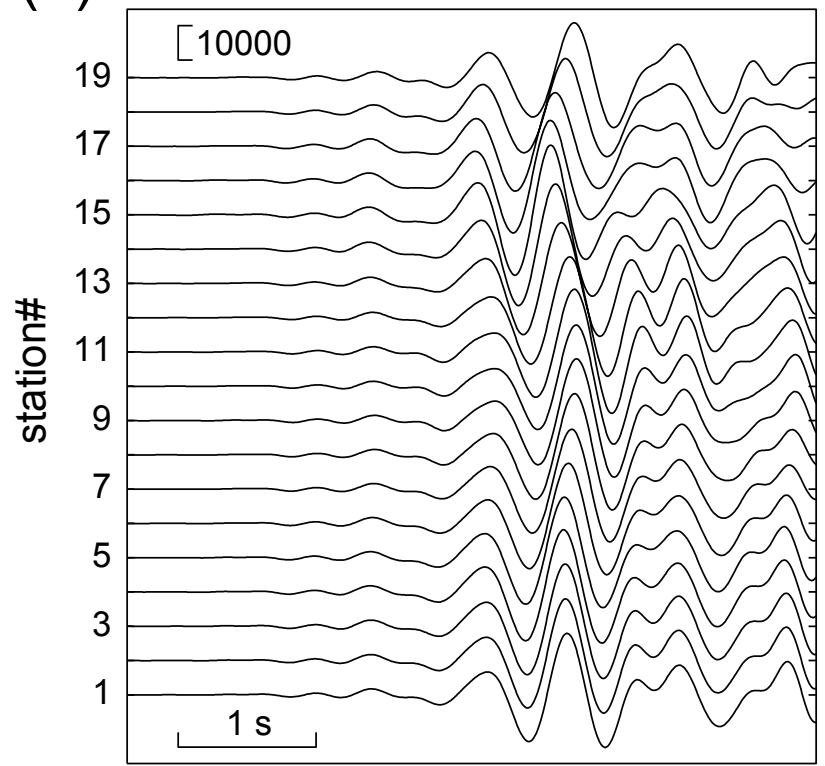

0 RMS 10000
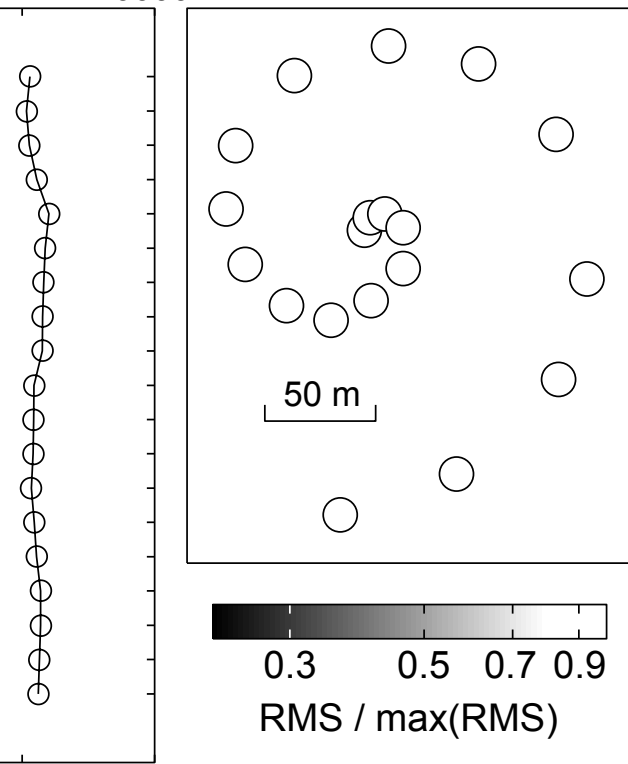


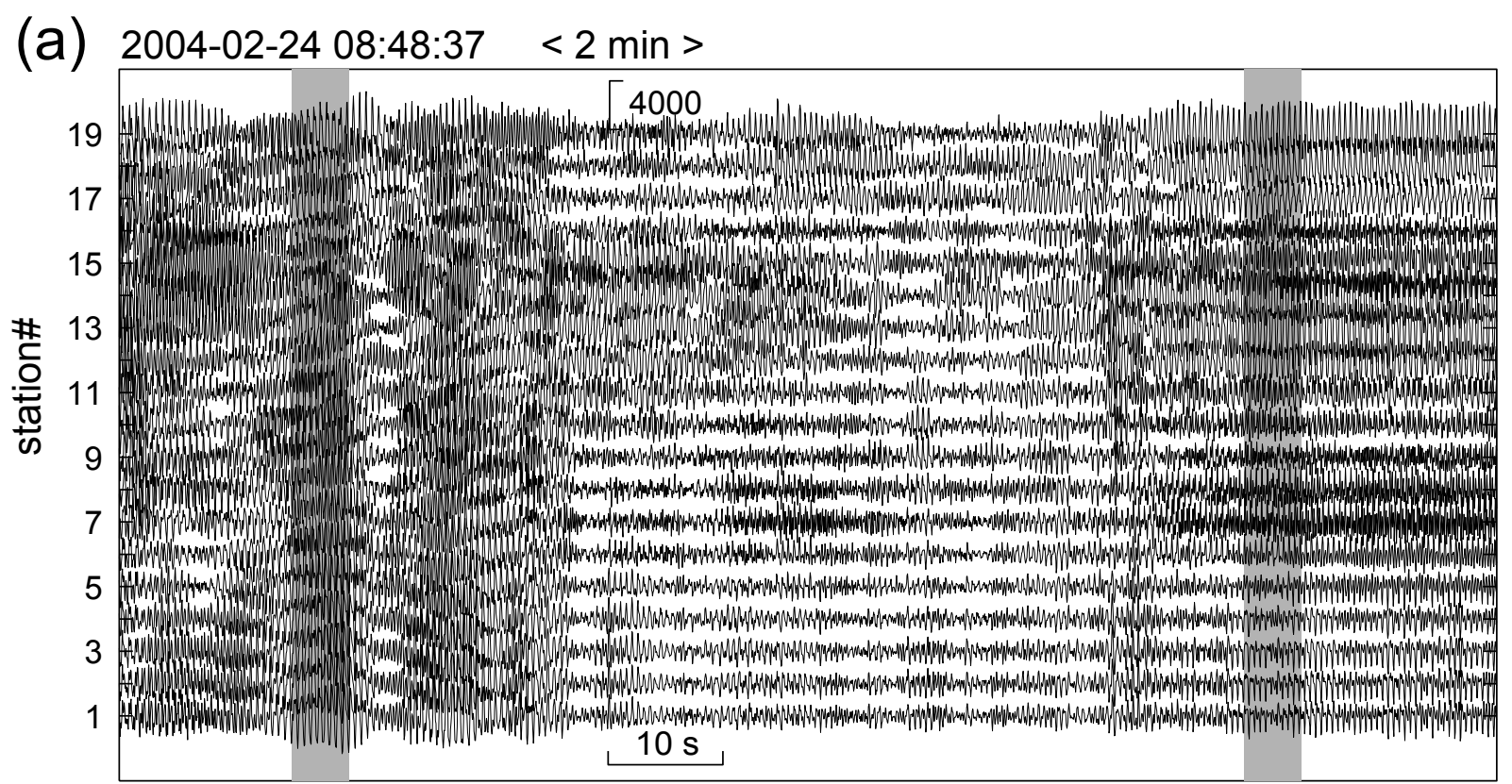

(b) 2004-02-24 08:48:52 <5s >

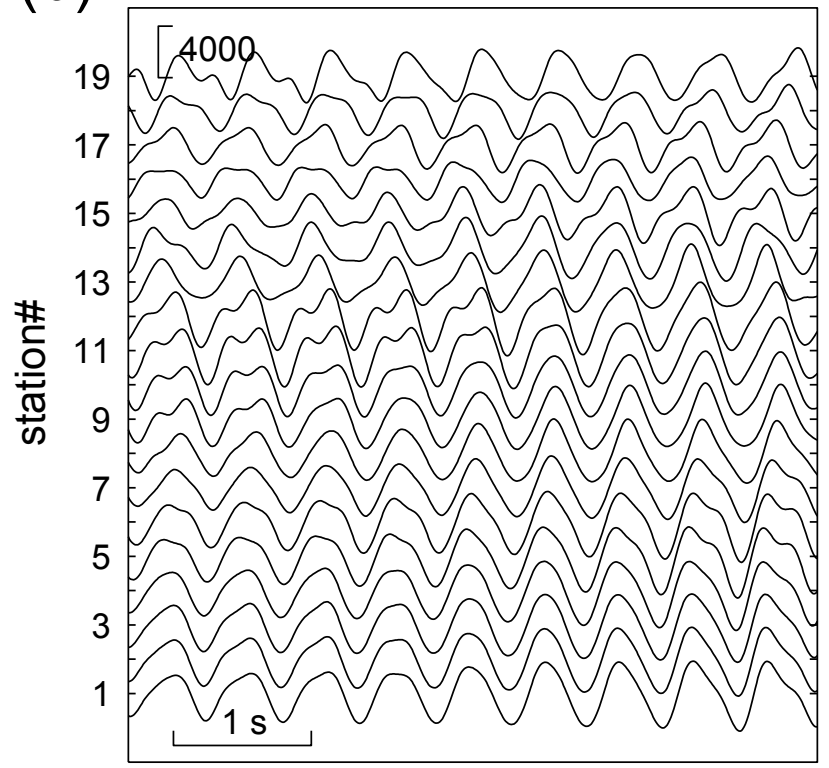

$0 \quad$ RMS 2000

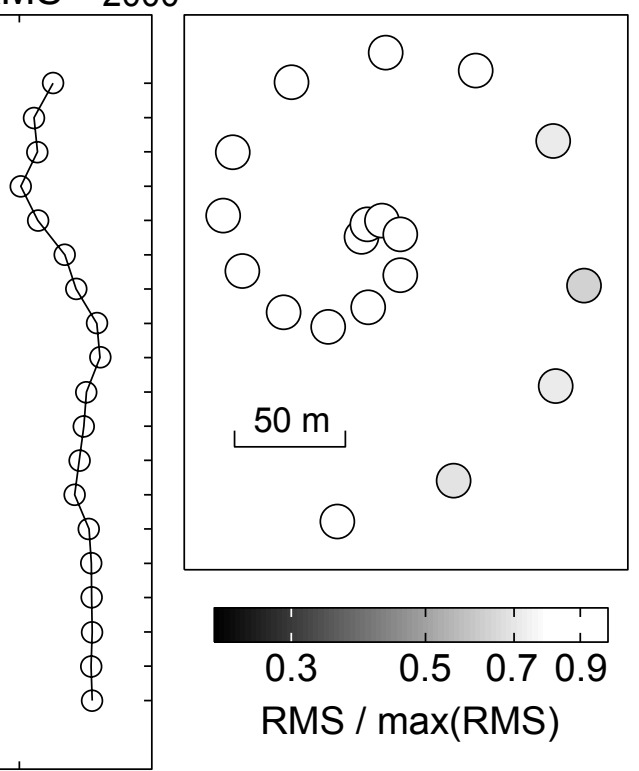

(c) 2004-02-24 08:50:15 $\langle 5 \mathrm{~s}\rangle$

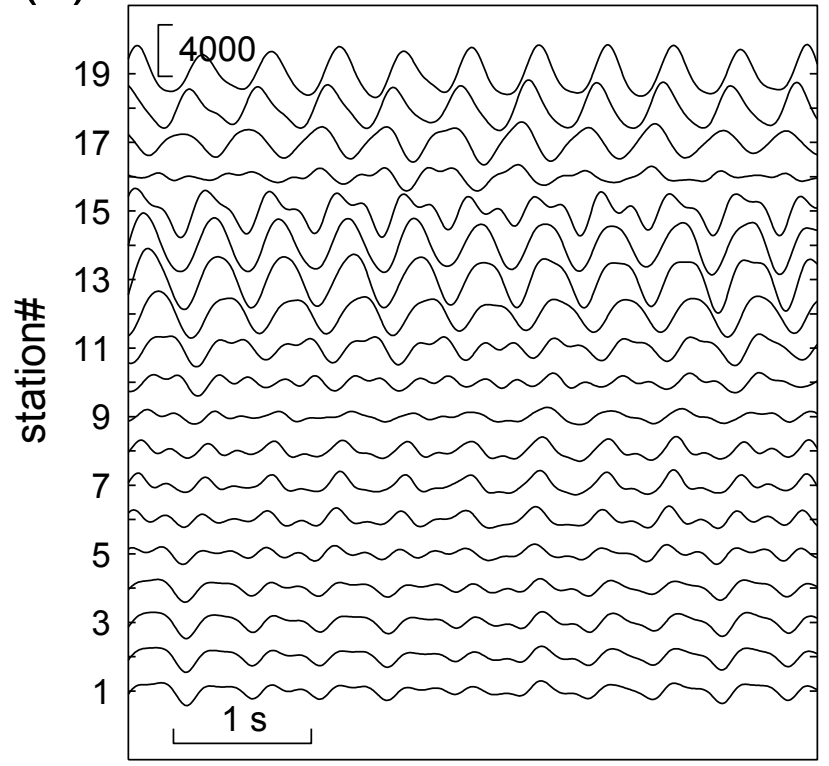

$0 \quad$ RMS 2000

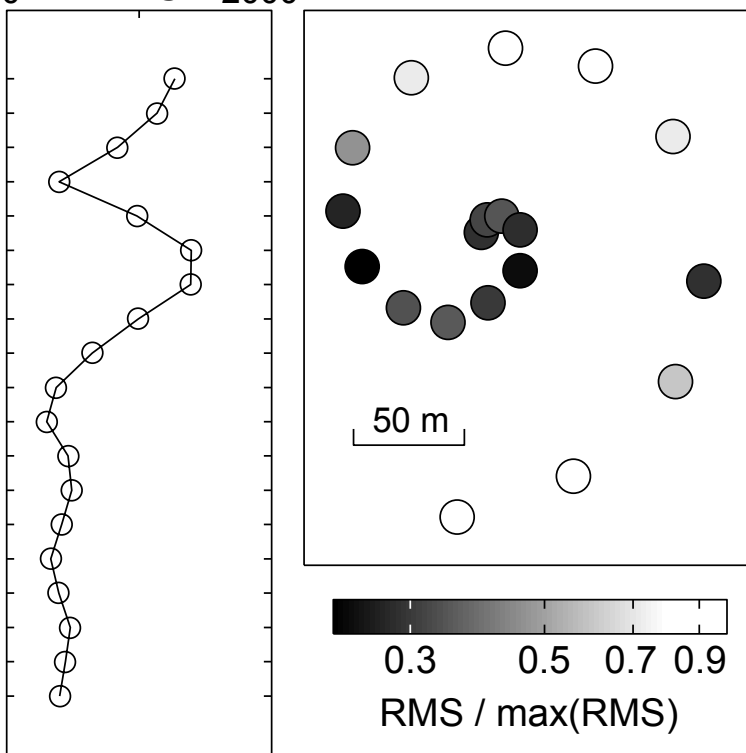



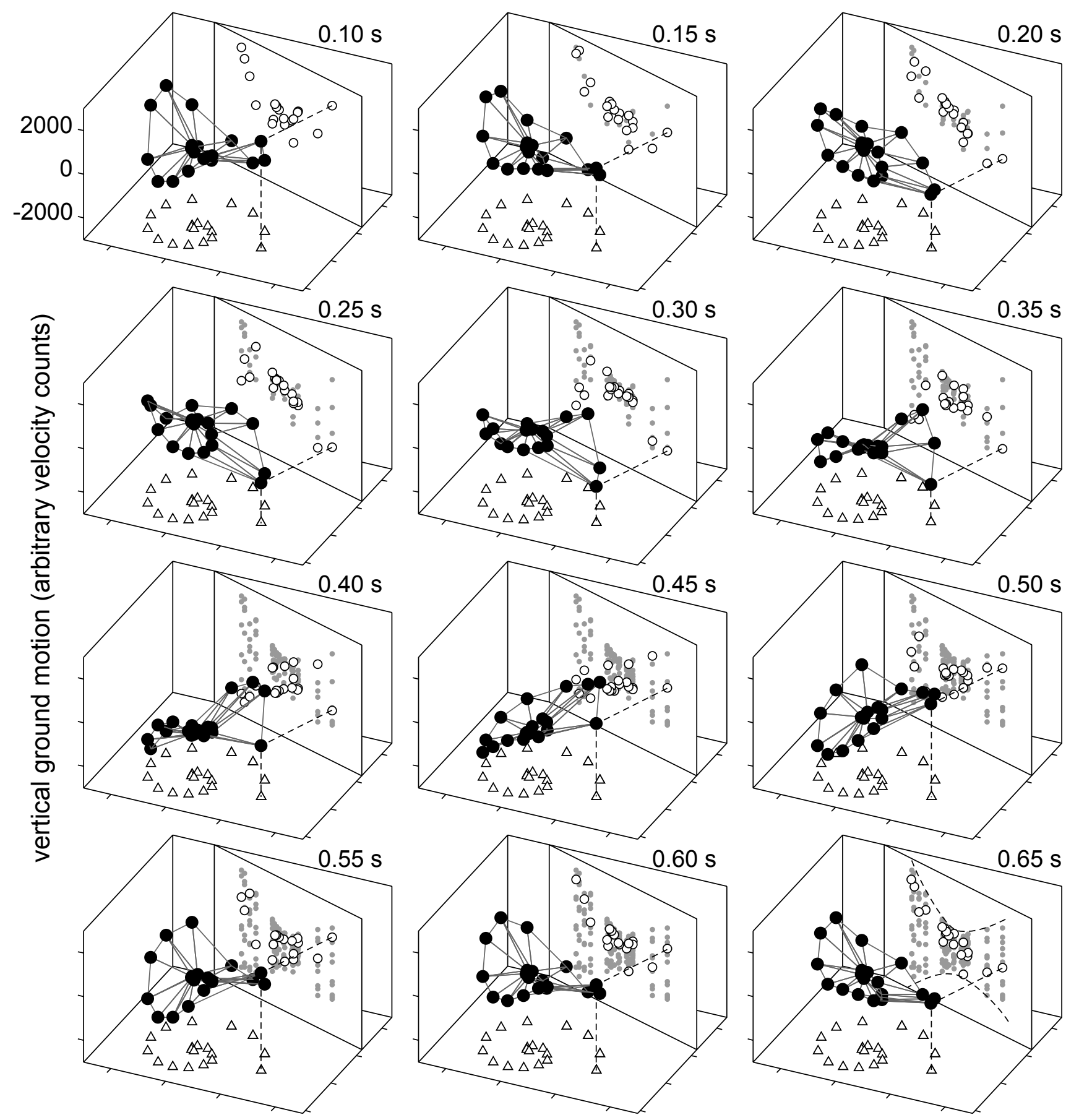

Almendros et al 2012 - Figure 4 

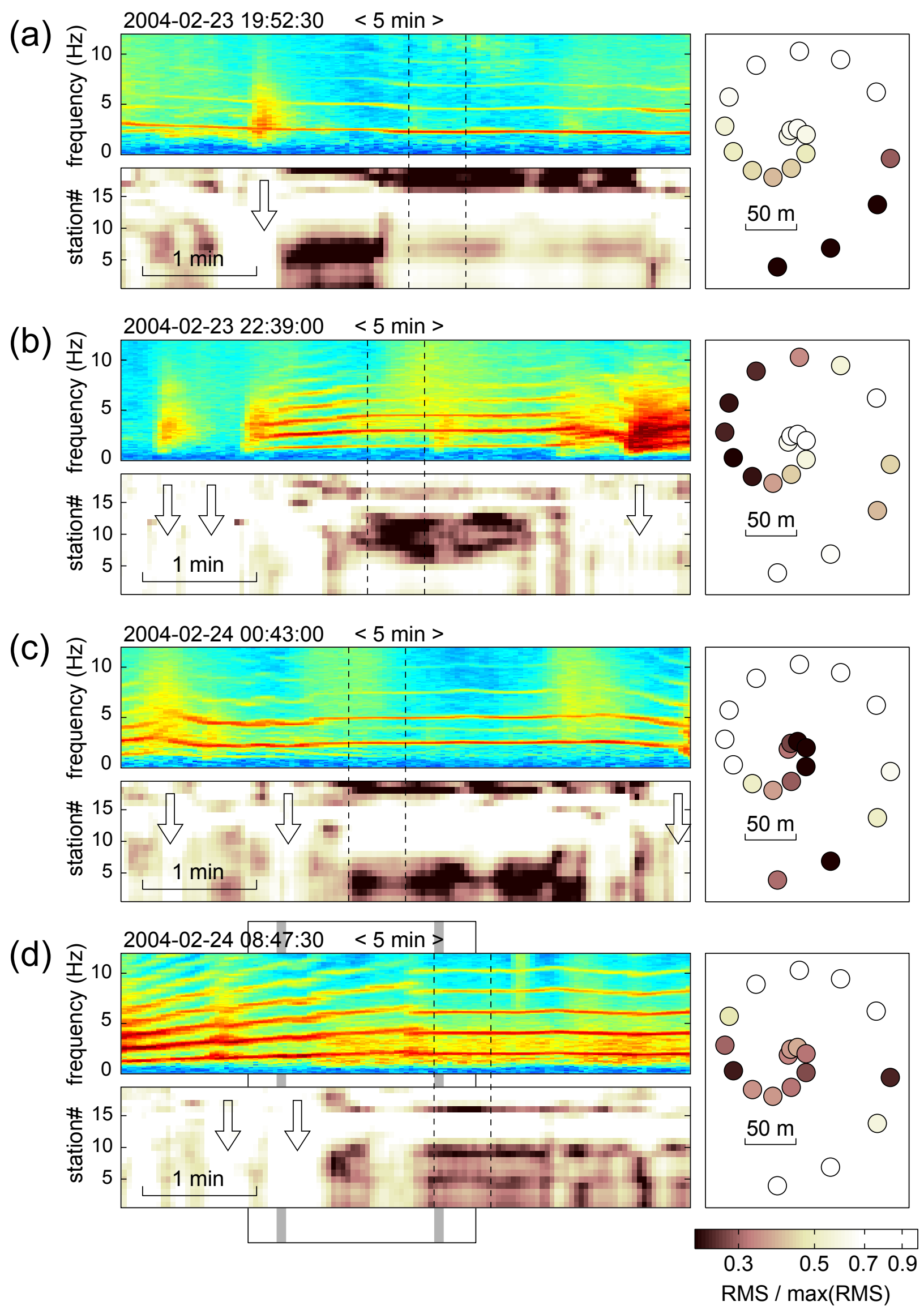


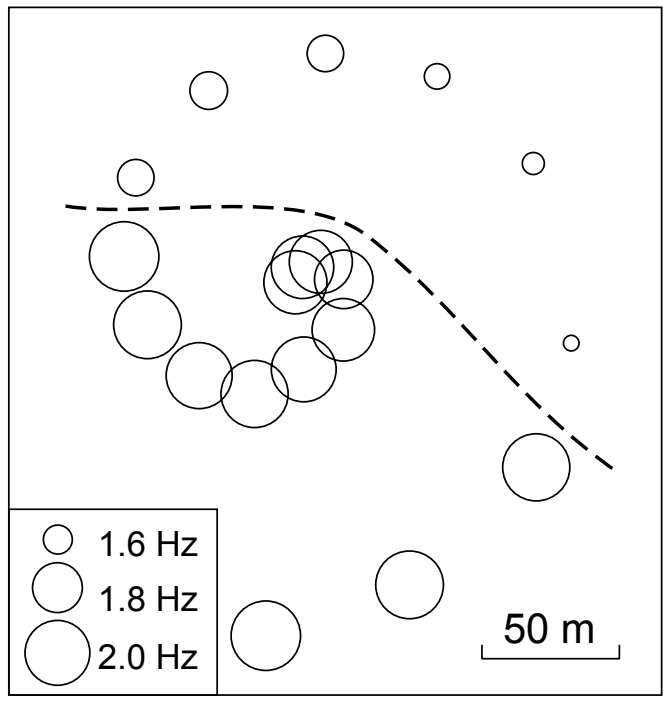

Almendros et al 2012 - Figure 6 


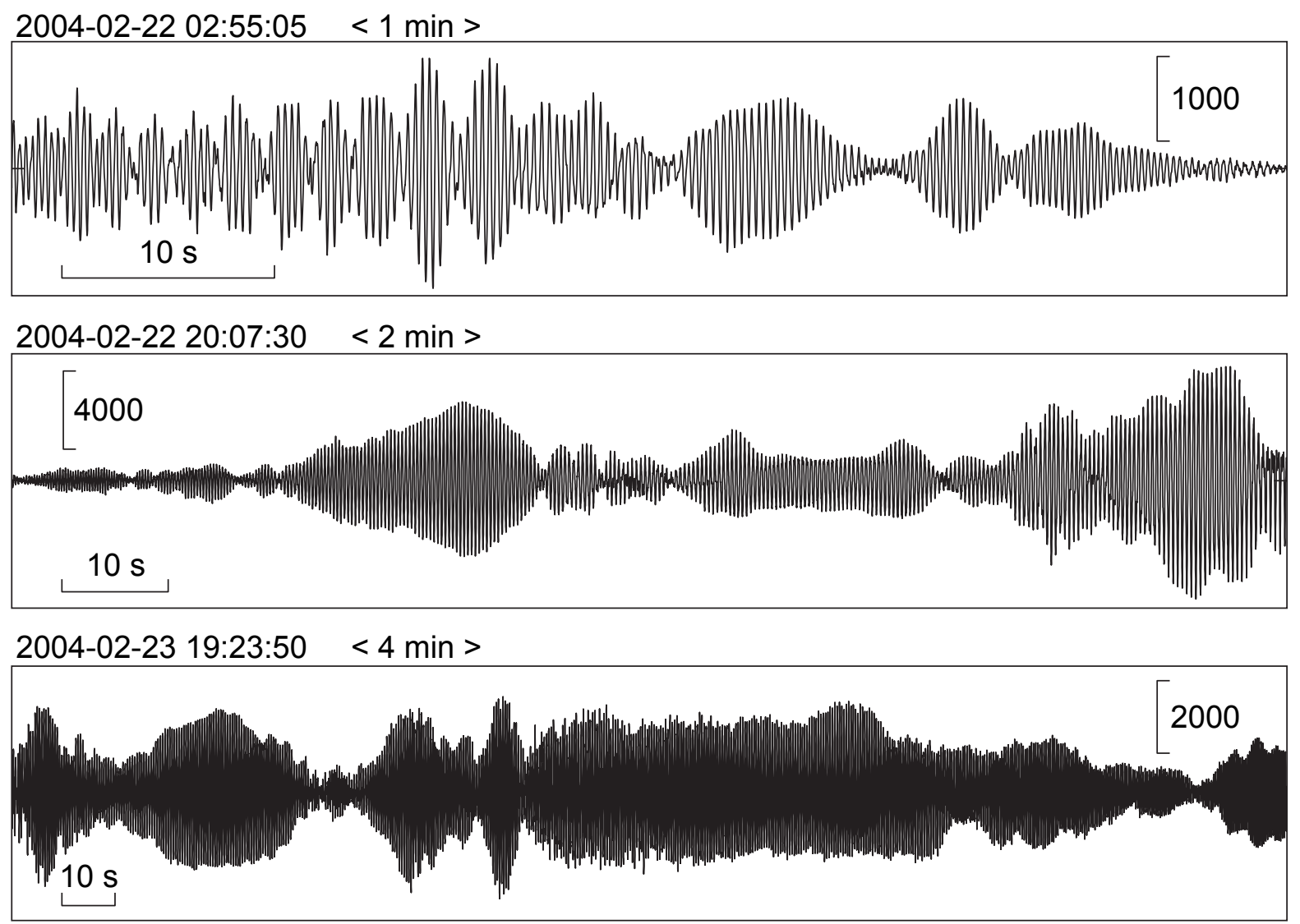

Almendros et al 2012 - Figure 7 
(a)
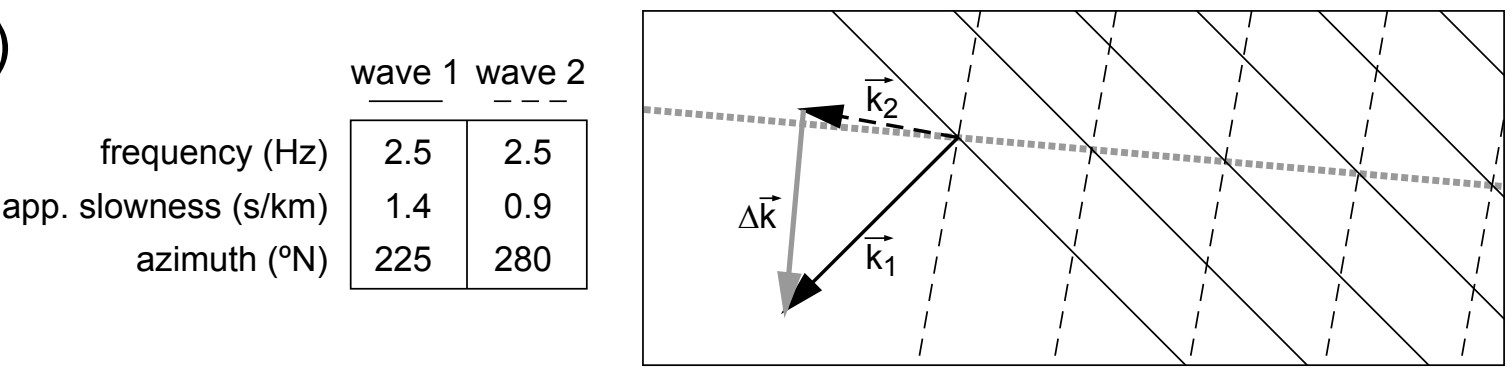

(b)
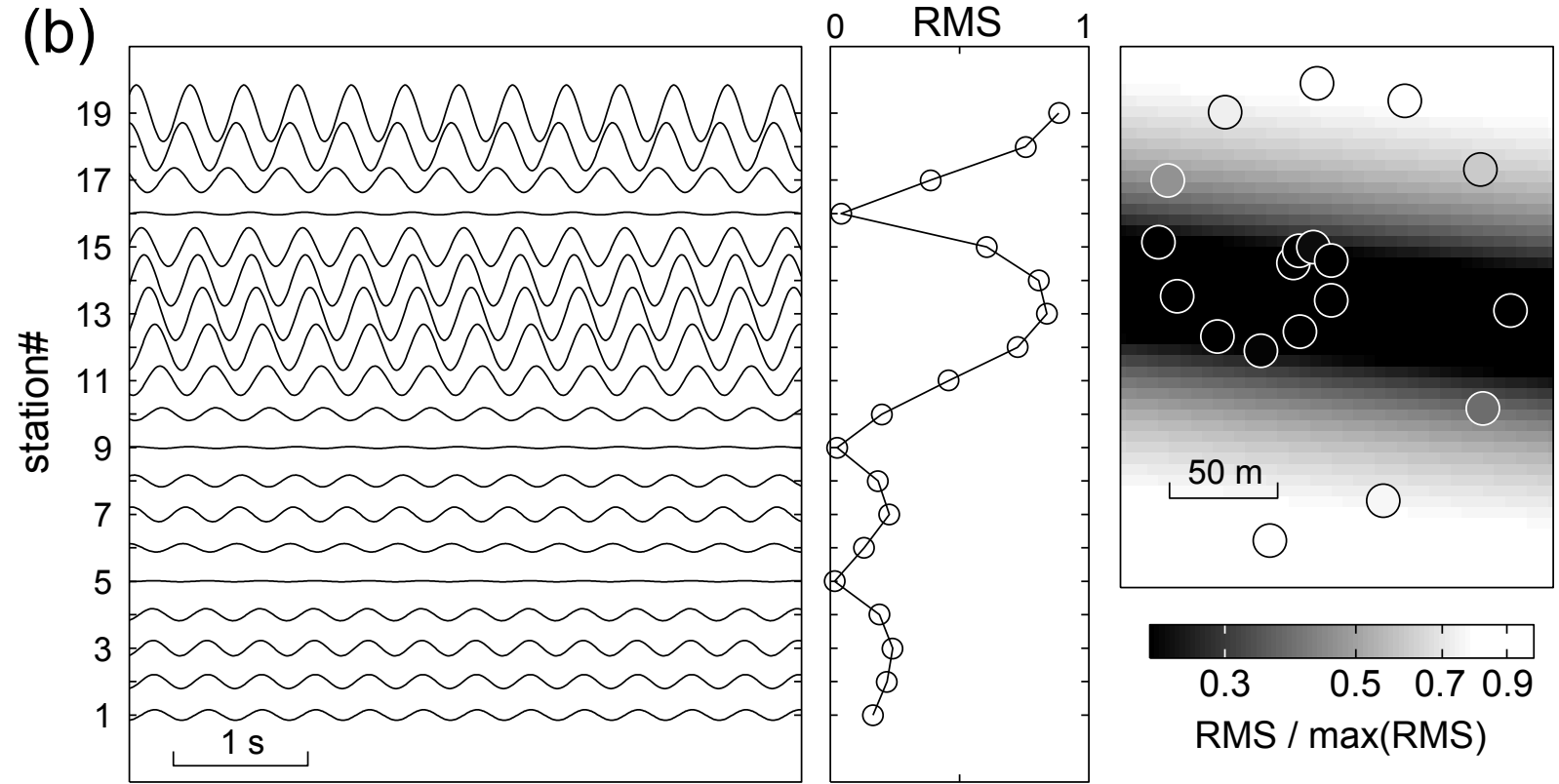

(c)
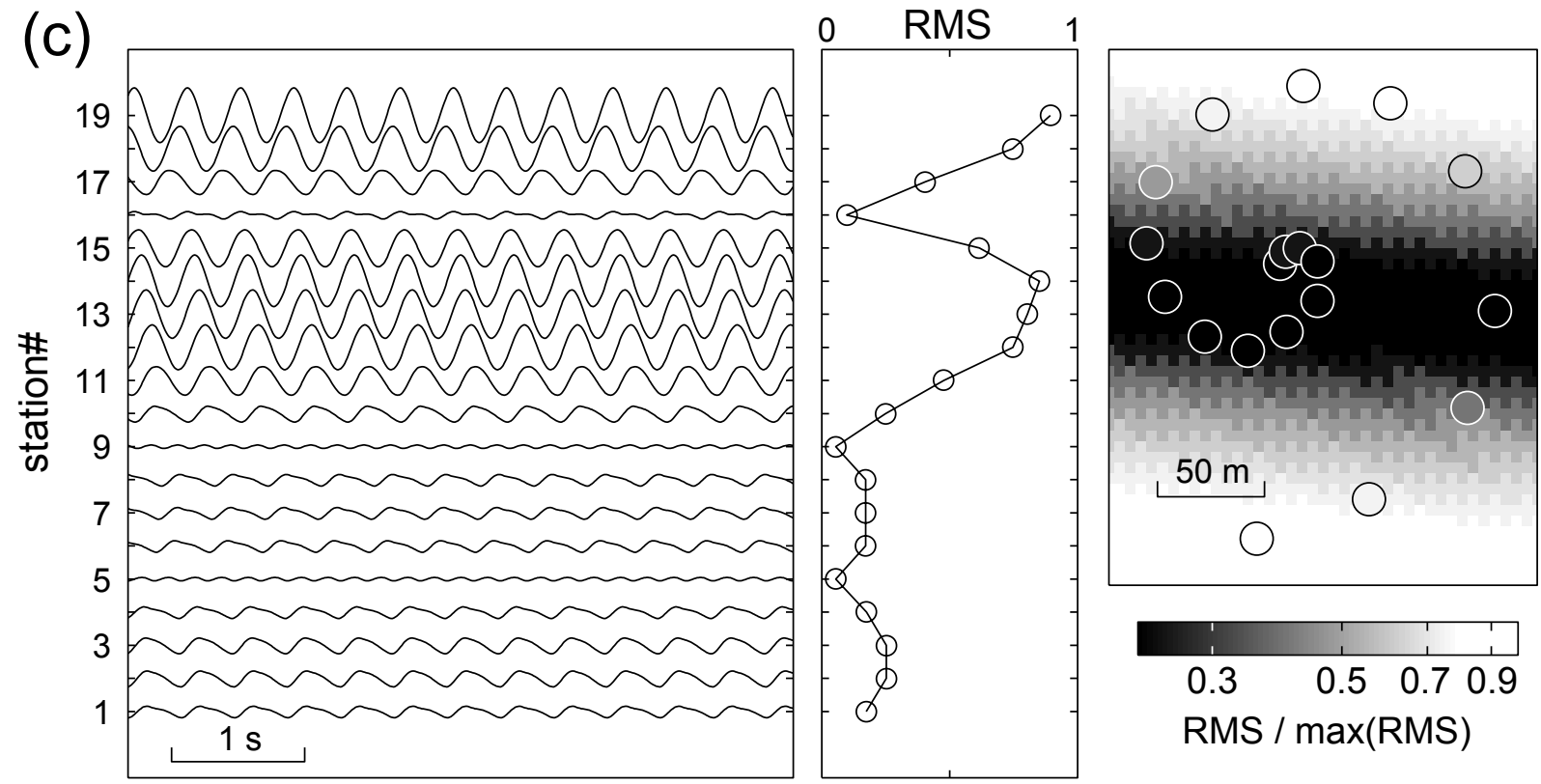

Almendros et al 2012 - Figure 8 
(a) 2004-02-24 08:48:37 <2 $\min >$

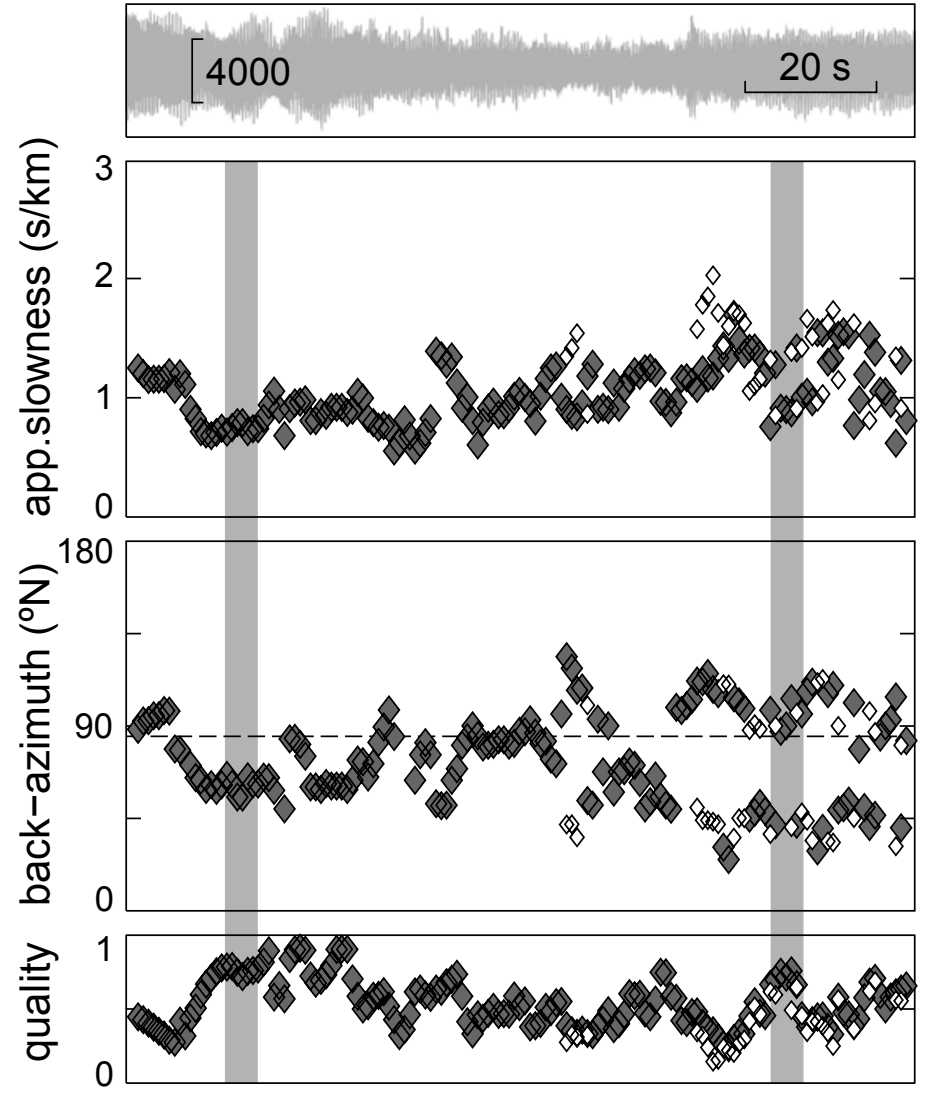

(b) 2004-02-23 14:04:40 <2 $\min >$ 2000 $20 \mathrm{~s}$
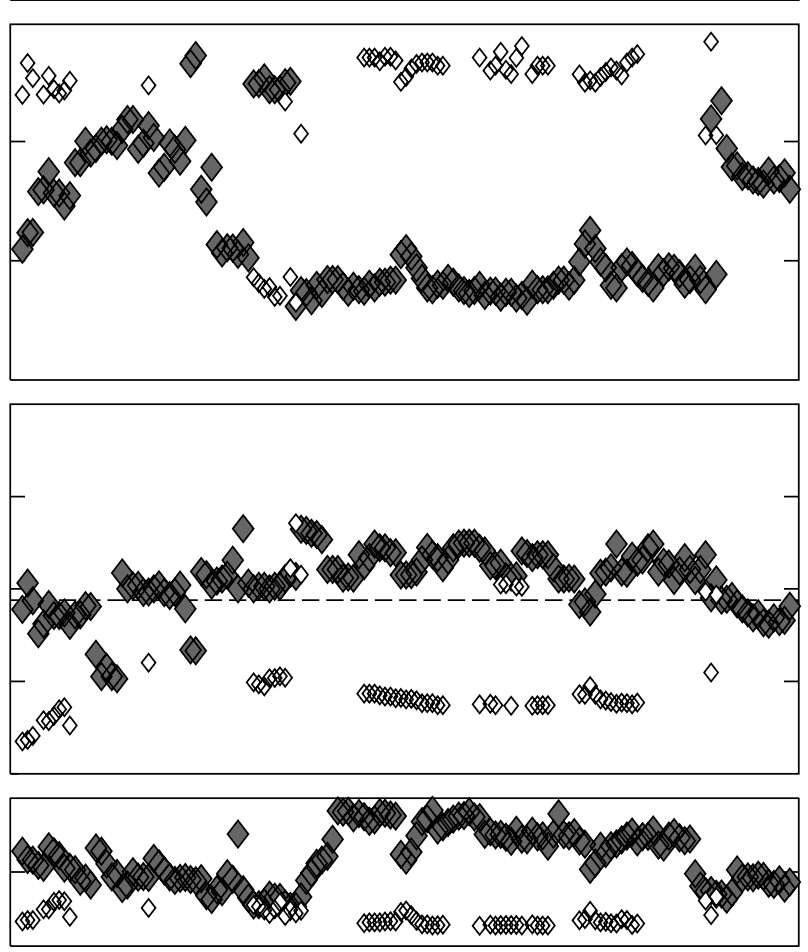